\title{
Investigating the Impact Of 5G Radiation on Human Health Using Machine Learning
}

\author{
O.E. Aru ${ }^{1}$, K.C. Adimora ${ }^{2}$,, F.J. Nwankwo ${ }^{3}$ \\ 1,2,3 Department of Computer Engineering, Michael Okpara University of Agriculture Umudike, Umuahia Abia State, NIGERIA.
}

\begin{abstract}
The advent of $5 G$ has improved greatly the speed of data transmission in wireless mobile technology. On the other hand, it has put society in suspense due to ailments that came along with its deployment. Many attributed the emission of $5 G$ radiation as the main cause of cancer today and that has led to the writing of this article paper. The research study employed a machine learning technique that is based on an artificial neural network in modeling the $5 G$ wireless technology. MATLAB, Simulink was used to analyze the absorption and penetration level of $5 G$ electromagnetic energy pattern into biological tissue Deoxyribonucleic Acid (DNA). Our research result revealed that the energy produced by $5 G$ radiation at the non-ionizing region of the electromagnetic spectrum is small and cannot break into the chemical bonds of biological tissue Deoxyribonucleic Acid (DNA) or cause changes to cells that will result in either cancer or viral disease.
\end{abstract}

Keywords: 5G Radiation, Machine Learning, MATLAB Simulink, Human Health, Non-ionizing Spectrum.

\subsection{INTRODUCTION}

The use of mobile wireless devices is continuously on the increase because of the need for internet data access. The need for reliable and fastest data access brought about emergence research for the development of $5^{\text {th }}$ generation mobile wireless technology. This is because $5 \mathrm{G}$ uses radio waves to deliver data at a faster rate than current mobile wireless technologies. From the technological point of view, the $5 \mathrm{G}$ mobile wireless network technology is situated on the non-ionizing region of the electromagnetic spectrum shown in Figure 1 and is developed for higher mobile data volume per area, the higher user data rate, higher number of connected mobile wireless devices, and to reduce latency in data delivery. Compared to the existing mobile wireless technologies, 5G technology is more widely applicable and reliable in terms of robust network connectivity and speed of data transmission. Despite the number of solutions offered by $5 \mathrm{G}$, it is still seen as a threat to human life because of the ailments that came along with the deployment of the 5G wireless technology. Also, the expansion of wireless technology broadband with the shortest wavelength and lower radio frequency of $5 \mathrm{G}$ technology highlights the concern that human health might be at risk. Although, it was claimed that $5 \mathrm{G}$ causes some ill-health which has not been verified.

It is only an assumption that the electromagnetic radiation emitted from $5 \mathrm{G}$ technology is harmful once

*Corresponding author (Tel: +234 (0) 807807 3414)

Email addresses: kyrimanjero@gmail.com

(K.C. Adimora), okezearu@gmail.com (O.E. Aru), _ijeomavictory93@gmail.com (F.J. Nwankwo), human beings are exposed to it through the use of their smartphones, computer devices, and other mobile wireless devices. Although, there are exposure limits adopted by Federal Communication Commission (FCC) in 1990 based on a behavioral change in rats exposed to Microwave radiation. The exposure limit was designed to protect humans from short-term heating risk due to Radiofrequency Radiation (RFR) exposure. Let see where the radio frequency is located in the electromagnetic spectrum and the nature of its wavelength and frequency as shown in Figure 1. The higher frequencies of Electromagnetic (EM) radiation, consisting of x-rays and gamma rays, are types of ionizing radiation. The Lower frequency radiation, consisting of ultraviolet (UV), infrared (IR), microwave (MW), Radio Frequency (RF), and extremely low frequency (ELF) are types of nonionizing radiation. Figure 1 depicted the electromagnetic spectrum.

In this research paper, we established a standard metric to investigate the impact of $5 \mathrm{G}$ radiation and its effect on human health. Equation (1) and (2) are formulated for analyzing radiation penetration into biological tissue of human beings upon being exposed to electromagnetic waves.

$E F R \propto \frac{1}{D P_{i b t}}$

The equation (1) is set to verify if the absorption pattern of $5 \mathrm{G}$ electromagnetic radiation into biological tissue is injurious or not. 
$E F R=n \frac{1}{D P_{i b t}}$

Where EFR represents Electromagnetic Frequency Radiation, $\mathrm{n}$ is the constant of proportionality,
$D P_{i b t}$ is the Depth of Penetration into the biological tissue. The purpose of this research paper is to investigate the impact of 5G wireless radiation on human health using Machine Learning (ML) approach that is based on artificial neural network.

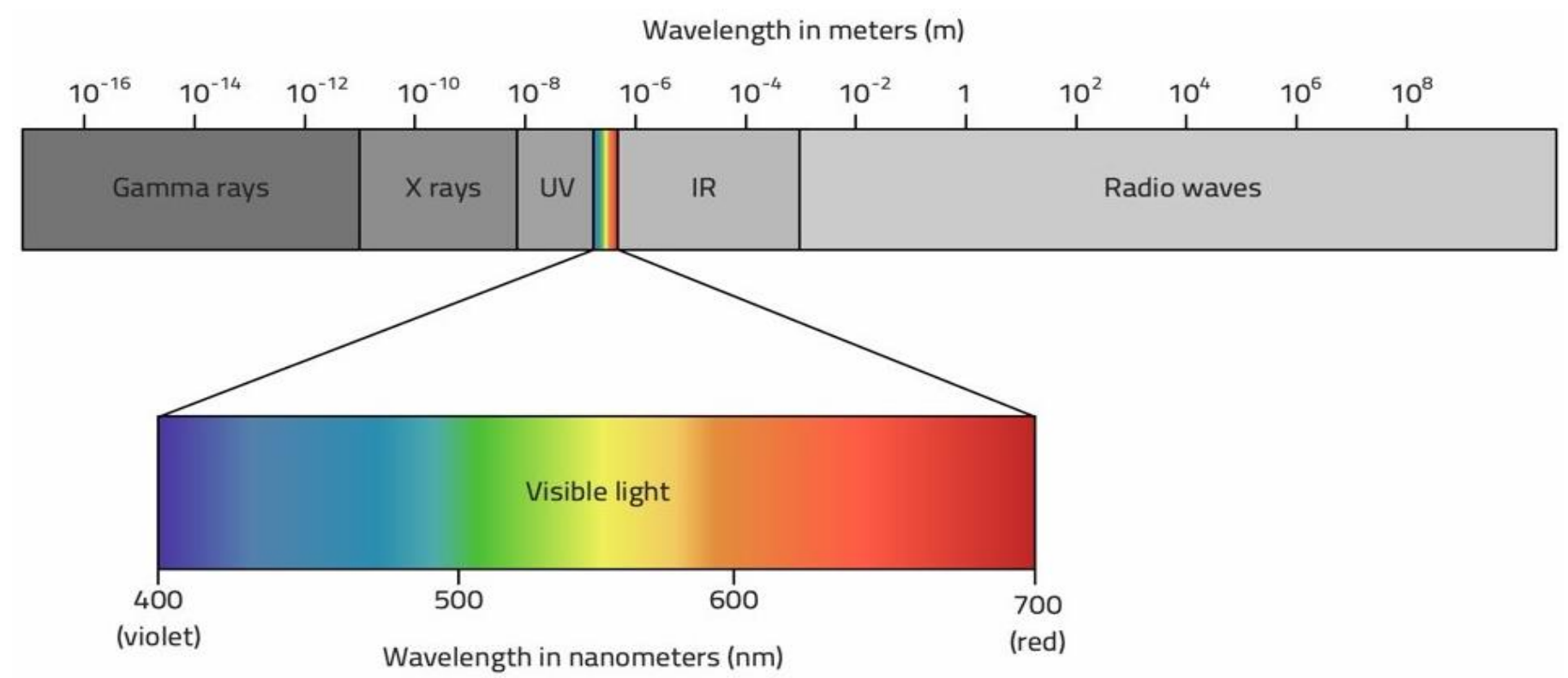

Figure 1: Electromagnetic Spectrum

The neural network model based on Spline function for measuring $5 \mathrm{G}$ radiation is designed as shown in Figure 2. In this paper, the theory of spline function in line with the machine learning approach is adequately combined with the neural network principle to achieve accurate precision of electromagnetic wave radiation that is measured by the weight $\omega_{n}(b)$ of neural network and spline function $\emptyset_{k}(a)$ is shown in Figure 2.

$$
\text { if } \sum \omega_{n}(b)+\emptyset_{k}(a)>0
$$

The radiation is harmful and must be avoided.

The summation of neural network weight and spline function analysis is detailed in the materials and method section of this research paper.

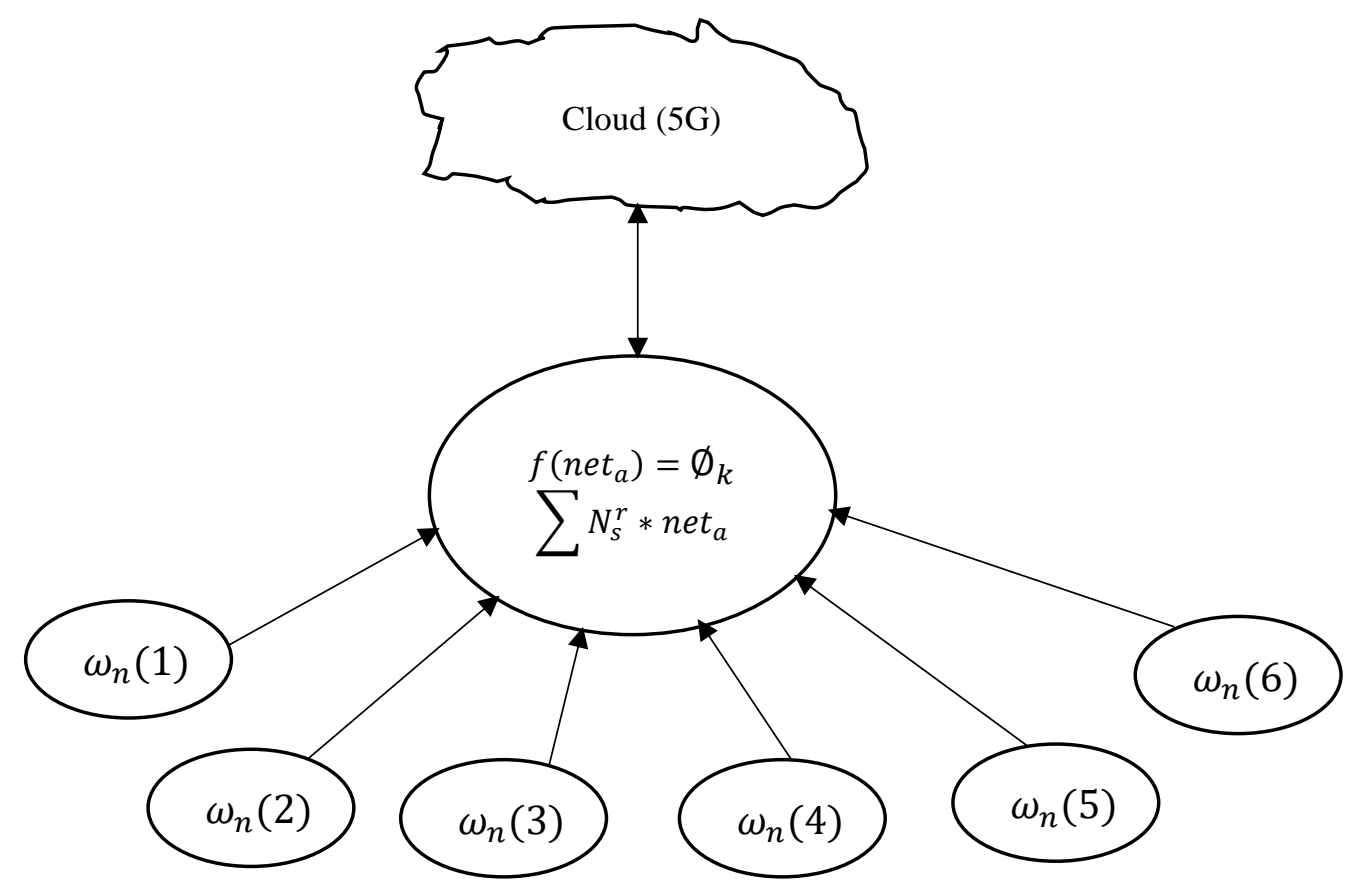

Figure 2: $5 \mathrm{G}$ Neural Network Model Based Spline Function 
Node Input $\omega_{n}(b)=\sum_{a} \omega_{(a b) n} * I_{a}$

Node Output $\emptyset_{k}=f\left(\right.$ net $\left._{a}\right)$

The neural network model shown in Figure 2 is based on machine learning with consideration of input and nodes in line with spline function requirements and specification. The cloud depicts the source of $5 \mathrm{G}$ signal transmission, and it is used in analyzing the electromagnetic radiation effect of $5 \mathrm{G}$ into biological tissue. Therefore, the spline function of the $5 \mathrm{G}$ network $\emptyset_{k}(n)$ is defined thus:

$\emptyset_{k}(n)=\left\{r^{a} a=0,1, \ldots, n ;\left(r-e_{l}-D_{i b t}\right) b=n+\right.$

$1, n+2, \ldots, f(N)\}$

Provided the nodal weight matrix $\omega_{n}(b)$ is not greater than zero $\left[\omega_{n}(b)\right]>0$ and $\left|\begin{array}{cc}-1 & 0 \\ 1 & -1\end{array}\right|<f(N)$ $\left[\omega_{n}(b)\right] *\left[\omega_{n}(b)\right]=\left[\omega_{0}, \omega_{1}, \omega_{2}, \ldots, \omega_{N-1}\right]$

Where $r$ is the network radiation; $e_{l}$ is the radiation emmision level;

$D_{i b t}$ is the depth of damage into biological tissue $f(N)$ represent the function of the $5 G$ netwrok

The cloud (5G) in Figure 1 above is on the electromagnetic spectrum which has two important regions. The ionizing and non-ionizing regions. The $5 \mathrm{G}$ technology is situated in the non-ionizing region of the electromagnetic spectrum and makes use of highfrequency millimeter wavelengths and the radiation from this region has low frequencies and bigger wavelengths though shorter in length. Therefore, it doesn't produce enough electromagnetic energy to break apart the chemical bonds of DNA or any biological tissue be it human or other animals. Prior to now, researchers have worked on many health related issues connected to $5 \mathrm{G}$ with the conclusion that, the non-ionizing region where $5 \mathrm{G}$ is located does not produce any energy capable of causing changes to cells that could lead to cancer or DNA mutation. In this research study, our major concern is to investigate the impact of $5 \mathrm{G}$ and also to verify the research on $5 \mathrm{G}$ related to health.

\section{$1.1 \quad$ Related Work}

Ruzat et al. [1] carried out a machine learning based technique research work for signal prediction to interference and noise ratio in mitigating the radio resource usage in mobile networks. The research paper, concentrated only on radio resource management and said nothing concerning the effect of radio radiation on human health.

Magnus [2] work on machine learning algorithm radio-based positioning to overcome signal obstructions to line of sights. The thesis work only addressed issue related to line of sight and said nothing to radio radiation to human health.

Natalia et al. [3] conducted a research on realizing quality of service through network slicing for heterogeneous communication services over common network infrastructure. The research study handled issue with network slicing and did not solve any problem related to electromagnetic radiation to human health.

Guto et al. [4] conducted research on a systematic review about how deep learning is being applied to solve some $5 \mathrm{G}$ issues. The shortcoming is that the work only concentrated to review work and did not provide any solution to the identified issues of 5G technology unlike the proposed system.

Scott et al. [5] conducted research overview of device-to-device (D2D) communications 5G heterogonous network by means of various Integer Linear programming. The study only handles issue related to $5 \mathrm{G}$ signal transmission using D2D communication principle and never said anything concerning the impact of $5 \mathrm{G}$ radiation on health matters.

Vila et al. [6] conducted research that examined the relation between occupational RF and Intermediate frequency (IF) EMF exposure and brain Tumor risk. According to the research results, exposure to RF electric and magnetic fields are suggestive of potential role in brain tumor promotion/ progression and should be further investigated. The shortcoming is that the work is inconclusive and is concentrated only on brain.

Verma [7] conducted research on the harmful effect of $5 \mathrm{G}$ radiation for various verticals such as health, environment issue, privacy, and thermal effects. The research shortcoming is that only review exercise was carried out and no implementation was done to solve the highlighted problems.

Simko [8] presented a call of research study to improve safety assessment of $5 \mathrm{G}$ radiation of local heat developments on small surfaces, such as the skin, eye, and environment. The paper is only for review exercise and no system was implemented to solve $5 \mathrm{G}$ radiation health problem.

\subsection{MATERIAL AND METHODS}

The materials and method used in this research

are:

Materials used are:

- MATLAB IDE

- 5G Wireless Module

- Smartphone Device

- Network Access Point

- Visual Studio for Python code implementation

\subsection{Machine Learning Neural Network based Algorithm}

In this section of the research study, different algorithms are developed for detailed of human beings 
and later modeled into two layer neural network. Analysis of emitted electromagnetic radiation from $5 \mathrm{G}$ technology. The Machine Learning Algorithm based on artificial neural network is developed for assessing the $5 \mathrm{G}$ absorption rate into biological tissue

\section{Algorithm Pseudo Code}

Input Layer $\boldsymbol{X}_{\boldsymbol{R} n}$ : Training data with parameter of emitted radiation

Number of Neuron Required for Training Data $(N)$

Training Instance out of all radiations instances applied to update

$$
\text { Weight } \omega_{n}(\varphi)
$$

Hidden Layer: $X_{R n}=\left\{X_{R 1}, X_{R 2}, X_{R 3}, \ldots, X_{R n-1}\right\}^{T} \in$ $R^{n * P l}$

\section{1: Encoder functionperformed the encoding of features}

$$
f(h)=\emptyset_{1}\left(X_{R n} \omega^{1}\right)
$$

2: Decoder that perform the function of reconstruction of feature (Radiation)

$$
X_{R n}=g(f(h))=\emptyset_{2}\left(f(h) \omega^{2}\right)
$$

\section{3: The output of decoder is equal to original} features Set of $5 G$ Radiation

Output Layer: $W_{n}(a z)$ : Feature Weight

1: $n \leftarrow$ Total Instances used for training

2: $R \leftarrow$ Feature Used

3: $W_{n}[a z] \leftarrow\left[\begin{array}{ll}1 & 0 \\ 0 & 1\end{array}\right]<0$

4: for $n \leftarrow 1$ To $N$ do

\section{5: Select randomly 'Radiation Target'Instance[BT]}

6: Compute heat of $5 G$ Radiation[H]and number of Neuron [N]

$$
\begin{aligned}
& \text { 7: for } \varphi \leftarrow 1 \text { To a do } \\
& \text { 8: } W_{n}[\varphi] \leftarrow W_{n}[a z]-\frac{\operatorname{Diff}\left(\varphi, R_{n}, H\right)}{n}+\frac{\operatorname{Diff}\left(\varphi, R_{n}, N\right)}{n} \\
& \text { 9: end for } \\
& \text { 10: end for }
\end{aligned}
$$

\section{1: Return $W_{n}[\varphi]$ : Feature Weight Vector $v($.$) that$ Compute depth of absortion}

The above algorithm is the subset of neural network mathematical representation of $5 \mathrm{G}$ electromagnetic radiation into biological tissue. The encoder and decoder process defined in the hidden layer section of the algorithm is automated. This is unsupervised learning model for the extraction of useful feature of the emitted $5 \mathrm{G}$ electromagnetic radiation. Let consider,

$X_{R n}=\left\{X_{R 1}, X_{R 2}, X_{R 3}, \ldots, X_{R n-1}\right\}^{T} \in R^{n * P l} \quad, \quad$ is unlabeled sample matrix where $n$ is the number of times electromagnetic radiation is emitted with penetration level (PL) dimension. The consideration of 5G neural network mathematical model is to let a network of neurons learn patterns related to electromagnetic radiation during a learning phase so that it is possible for the network to classify new input data after the learning phase.

In the $5 \mathrm{G}$ pattern recognition, in line with neural network defined mathematical model, a feed-forward neural network model that is based on a combination of a linear and non-linear parametric function is defined. We used a two layer neural network with adjustable weights in the analysis of the algorithm expressed above. When the summation of adjustable weights is more than zero it is said to fire and at that time the radiation from $5 \mathrm{G}$ technology can cause changes to biological cells that will lead to either cancer or virus disease. The Machine Learning adjustable weights based on neural network is formulated thus:

$$
\begin{aligned}
& \sum_{R=0}^{N+M L} W_{n}(a z)=\omega_{n+0}(a * b)+\omega_{n+1}(c * d)+ \\
& \omega_{n+2}(e * f)+\cdots+\omega_{n+N}(y z)^{N}
\end{aligned}
$$

Therefore, the adjustable weights in the form of $\omega_{n+N}(a b)^{N}$, when $n \neq 0$, are referred to as weights and $W_{n}(a b)$ is taken as biases. The weights are adjusted by optimizing a predetermined the absorption function of $5 \mathrm{G}$ radiation. The two layer neural network diagram shown in Figure 3 describes radiations input with weights into human biological tissues at different layers denoting the chemical bonds of DNA or biological tissues.

The variables in Figure 3 are defined as follows: $x_{R n}$ is the $5 \mathrm{G}$ Emitted Radiation Input Layer (BT1)is the First Biological Tissue Hidden Layer (BT2) is the second Biological Tissue Output Layer (BT3)is the Third Biological Tissue $\omega_{n}(\varphi)$ is the Adjustable Weights that determines the $5 \mathrm{G}$ radiation impact.

$y_{n c 1}$ is the output result revealing no cancer when $y_{n c 1}$ is $\leq 0.5$

Note: when any of the DNA chemical bond indicates red color, it implies that the $5 \mathrm{G}$ emitted radiation is harmful and can cause changes to the biological cells that will lead to either cancer or viral disease. In our study sample all the layers are green in color and are represented with vertices which implies that the emitted radiations have no negative impact on human health. Also, the weights are represented with edges.

In the Figure 3, the hidden layers model of the biological DNA allow for the function of a neural network to be broken down into specific transformations of the 
electromagnetic radiation targeted on humans so that the defined output of electromagnetic energy from the hidden layer will not be enough to break into the biological bonds of the DNA to cause any ill-health or viral disease. Each hidden layer function is specialized to produce a defined output.

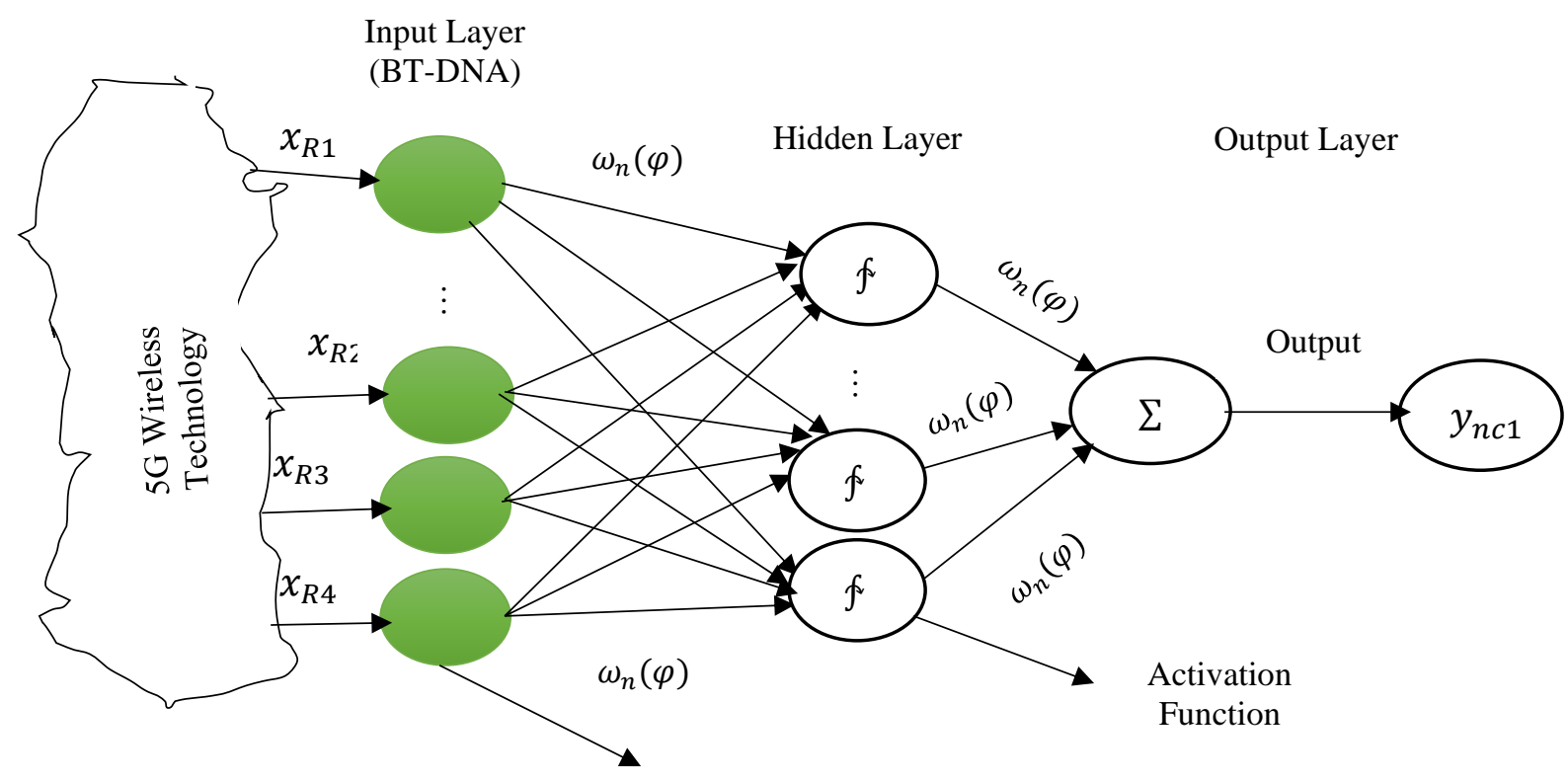

DNA Chemical Bond

Figure 3: Neural Network Modeling of 5G Radiation

For the Feed-Forward calculation of this research study, the hidden layer node of the biological DNA is formulated thus:

Hidden Layer $(H L)=\sum$ Input Layer $(I L) *$ Assigned Weight (AW)

Bias weight was not used in the design of the neural system for $5 \mathrm{G}$ radiation analysis. Therefore,

$$
H L 1=\sum_{1=0}^{n} \omega_{n}(\varphi) * I L_{1}+\omega_{n}(\varphi) * I L_{2}
$$

If the value of the hidden layer is $(H L)>0.5$ then $5 \mathrm{G}$ electromagnetic wave (radiation) is said to fire but would not penetrate the biological tissue or cause DNA mutation unless the hidden layer value is equal to 1 which is technically impossible. The research study ensured that each node in the hidden layer undergoes the activation function calculations and the activation function used in this research study is a Sigmoid S-Shape function that processed the transformed radiation data by hidden layer into output as stated in equation (9).

On a general form the two-layer neural network is given by equation (8).

$Y_{n c}\left(x_{R n}, \omega_{n}(\varphi)\right)=v\left(\sum_{n=0}^{N} \omega_{n}(\varphi) * h\left(\sum_{a}^{R} \omega_{n}(\varphi) *\right.\right.$

$\left.\left.x_{R 1}+\sum_{a}^{R} \omega_{n}(\varphi) * x_{R 2}\right)+\omega_{n}(\varphi) * R_{n}\right)$
Where $x_{R}=\left\{x_{R n}\right\}, n=1,2, \ldots . R$ is the input variable, and $Y_{n c}$ is the output variables controlled by the vector $\omega_{n}(\varphi)$ of adjustable weights given in (3). The transformation from the first layer to the hidden layer and from the hidden layer to output layer are done by differentiable, non-linear activation functions $h($.$) and v($.$) , respectively. In this paper we used$ sigmoid activation function to enable we analyze the adjustable weight of radiation properly. Hence $v(\alpha)$ is written thus:

$v(\alpha)=\frac{1}{1+e^{-i}}$

Where $v(\alpha)$ is the Hidden Layer Output $\left(H L_{\text {output }}\right)$ and $i$ is the Hidden Layer Input $\left(H L_{\text {input }}\right)$.

The results of Hidden Nodes, Hidden after Activation, Calculated Output, and Output after Activation are reported in results and discussion of Table 2.

The Figure 3 is the network diagram for a two-layer neural network with $\mathrm{N}$ neurons per hidden layer, $\mathrm{R}$ input variables and nc output variables. The sigmoid activation function is used with hidden layer and output layer. While weight parameters are defined thus:

$W_{n}(a z)=\left\{\omega_{1}(\varphi), \omega_{2}(\varphi)\right\}$ and $\omega_{n}(\varphi)$ represents the connection parameter between the input, hidden, and output layer. 


\subsection{Mathematical Model for Human Radiation Absorption}

The model for analyzing device -to-human signal transmission with electromagnetic radiation are stated thus. The 5G electromagnetic energy absorption rate at each hop on a device and human is computed as:

$$
\begin{aligned}
& A_{r}(H)=\frac{1}{\lambda} \sum_{(n, r) \in E W}^{N} \eta\left[\frac{\lambda_{n r} * e^{-i \omega t}}{\phi_{n r}-\lambda_{n r}}\right]\left[n \frac{1}{D P_{i b t}}\right]+ \\
& {\left[\frac{D_{r^{*} \operatorname{Cos}} \omega / 2}{\sin ^{2}(M)}\right]}
\end{aligned}
$$

Where, $A_{r(H)}$ is the Absolution rate of $5 \mathrm{G}$ radiation by human being.

$\lambda$ is the wavelength of $5 \mathrm{G}$ Electromagnetic wave; $(n, r)$ is emission rate.

$\varnothing$ is the angle of radiation transmission; $e^{-i \omega t}$ is time delay in penetrating the human skin.

$D P_{i b t}$ is the depth of penetration into biological tissue; $M$ is the medium of transmission.

$\omega$ is the speed of signal transmission; $D_{r}$ is the data rate. $\eta$ is the biological tissue protection efficiency.

Therefore, for a very close signal transmission, the impact of radiation can be modeled using fractional Brownian motion queuing system stated in equation (11).

$A_{r}(H)=\tau * \frac{1}{\lambda} \sum_{(n, r) \in E W}^{N}[\eta]\left[\frac{\lambda_{n r^{*} e^{-i \omega t}}}{\left(\phi_{n r}-\lambda_{n r}\right)^{\wedge} 2}\right]^{\frac{T}{4(n, r)-R}}$
Where $k(R)=R^{r}(1-R)^{1-R}, R \in[0.5,1] \quad$ is Hurst parameter, $r$ is the index of $5 \mathrm{G}$ radiation dispersion from the spectrum presented in equation (12).

$r=\bigsqcup \tau\left(1+\frac{T}{4(n, r)-R}\right)\left[2 K^{2}(R) a\right]^{\frac{T}{4(n, r)-R}}$

\subsection{RESULTS AND DISCUSSION \\ 3.1 The 5G Radiation Model of Neural Network}

The $5 \mathrm{G}$ radiation model of the neural network used in this research study is implemented in MATLAB with the help of the artificial neural network toolbox embedded in MATLAB IDE. The electromagnetic wave radiation of $5 \mathrm{G}$ is translated into a regression problem where the main goal is to fit a function from the input layer to the target biological tissues. The neural network used in this study have two hidden layer and activation function $h$ (.) that in the first layer is tanh (), and the activation function in the second layer is a pure line. The pure line implies that the electromagnetic radiation directed to human was not absorbed into the biological tissue or bring any change to the cells that might lead to cancer disease. Consider a data set containing radiation temperature of $5 \mathrm{G}$ technology reading that are measured every three hours. Interpolating the time of electromagnetic wave exposure to human and the corresponding temperature values. Table 1 is the $5 \mathrm{G}$ radiation experiment result that analyzed the impact of electromagnetic wave on humans.

Table 1: Data set from 5G Radiation

\begin{tabular}{ll}
\hline Time [Hours] & Temperature [Centigrade] \\
\hline March 18,00: 00 & 31 \\
March 18,03: 00 & 25 \\
March 18,06:00 & Nil \\
March 18,09:00 & 24 \\
March 18,12:00 & 36 \\
March 18,15:00 & 35 \\
March 18,18:00 & 33 \\
March 18,21:00 & Nil \\
March 19,00: 00 & 31 \\
\hline
\end{tabular}

The Figure 4 presented is the graphical representation of the temperature generated through $5 \mathrm{G}$ exposure to humans.

In Figure 4, the generated temperature from the electromagnetic energy of $5 \mathrm{G}$ never exceeded the room temperature $36^{\circ} \mathrm{C}$ and it is a proof that the heat from its radiation is incapable of breaking the chemical bond of biological DNA or cause any changes to the cells that can either lead to cancer or viral disease. At 18, 06: 00 and 18, 21:00 no temperature heat was recorded this is because of the short wavelength of $5 \mathrm{G}$ technology and its location in the non-ionizing region of the electromagnetic spectrum. Figure 5 depicts the 5G Electromagnetic Radiation Analysis. 


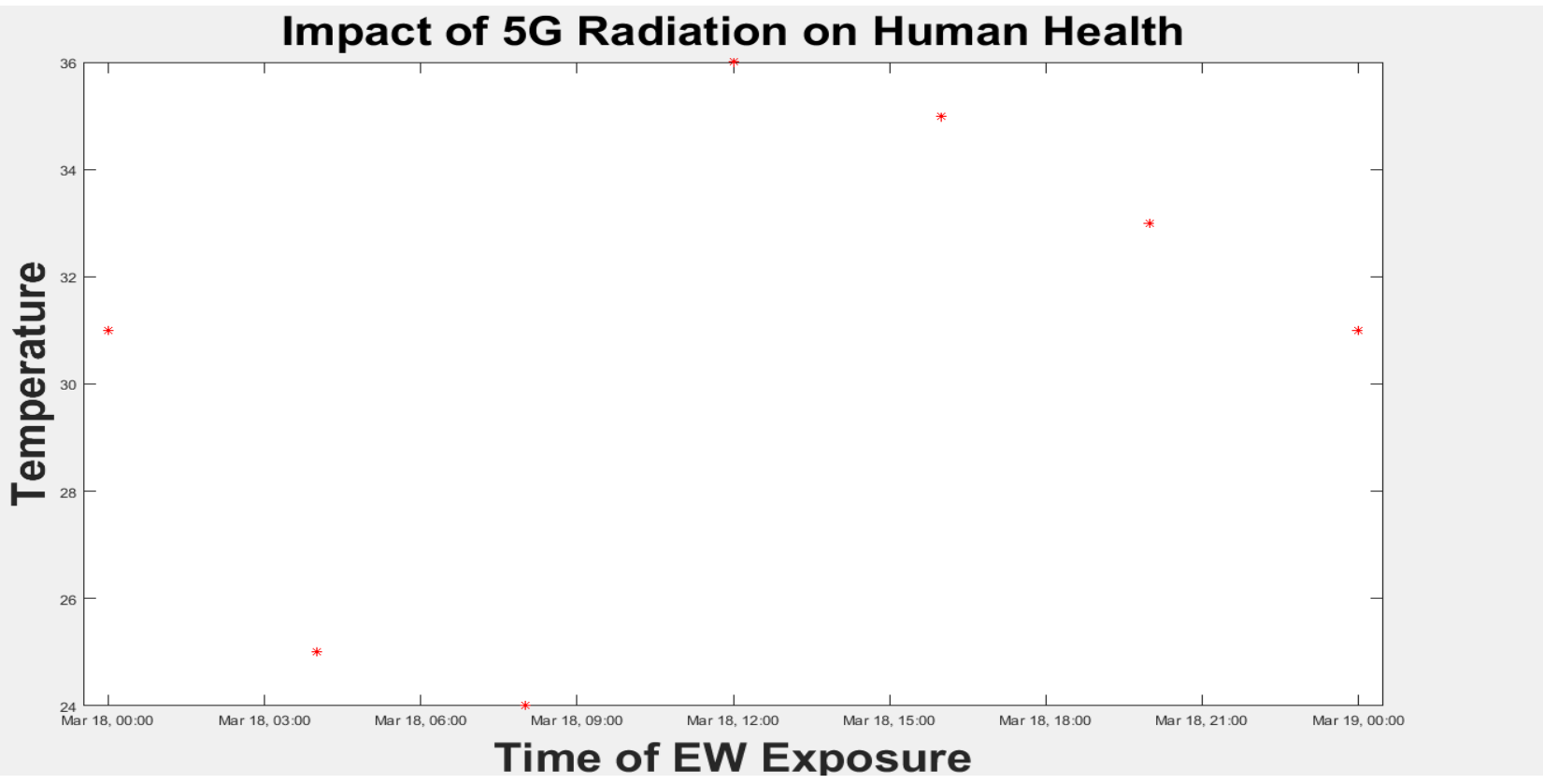

Figure 4: Analysis of $5 \mathrm{G}$ Radiation on Human Health

The Table 2 is the Hidden Nodes, Hidden after Activation, Calculated Output, and Output after Activation results from the hidden layer and sigmoid activation function formulated in material and methods of this study are tabulated and presented in Table 2.

The HL-output (HaA) values are less than 0.5 which implies no destructive harm to biological DNA,
OaA values are also less than 0.5 which confirmed that radiation targeted to humans from $5 \mathrm{G}$ is harmless and causes no change of cells that will lead to any biological tissue damage or ill-health. The values of the required output nodes are at optimal level and produces energy very low to break apart the chemical bond of biological DNA in human being.

Table 2: 5G Radiation Hidden Layer Result

\begin{tabular}{llllll}
\hline $\begin{array}{l}\text { Hidden } \\
\text { (HN) }\end{array}$ & Node & $\begin{array}{l}\text { Hidden after } \\
\text { Activation (HaA) }\end{array}$ & $\begin{array}{l}\text { Calculated } \\
\text { Output (CO) }\end{array}$ & $\begin{array}{l}\text { Output after } \\
\text { Activation (OaA) }\end{array}$ & $\begin{array}{l}\text { Output } \\
\text { (Required) }\end{array}$ \\
\hline HL1: 0.314 & HL-output 1: 0.463 & CO 1: 1.038 & OaA1: 0.451 & ON 1: 0.03 \\
HL2:0.346 & HL-output 2: 0.452 & CO 2: 1.121 & OaA 2: 0.462 & ON 2: 0.34 \\
\hline
\end{tabular}

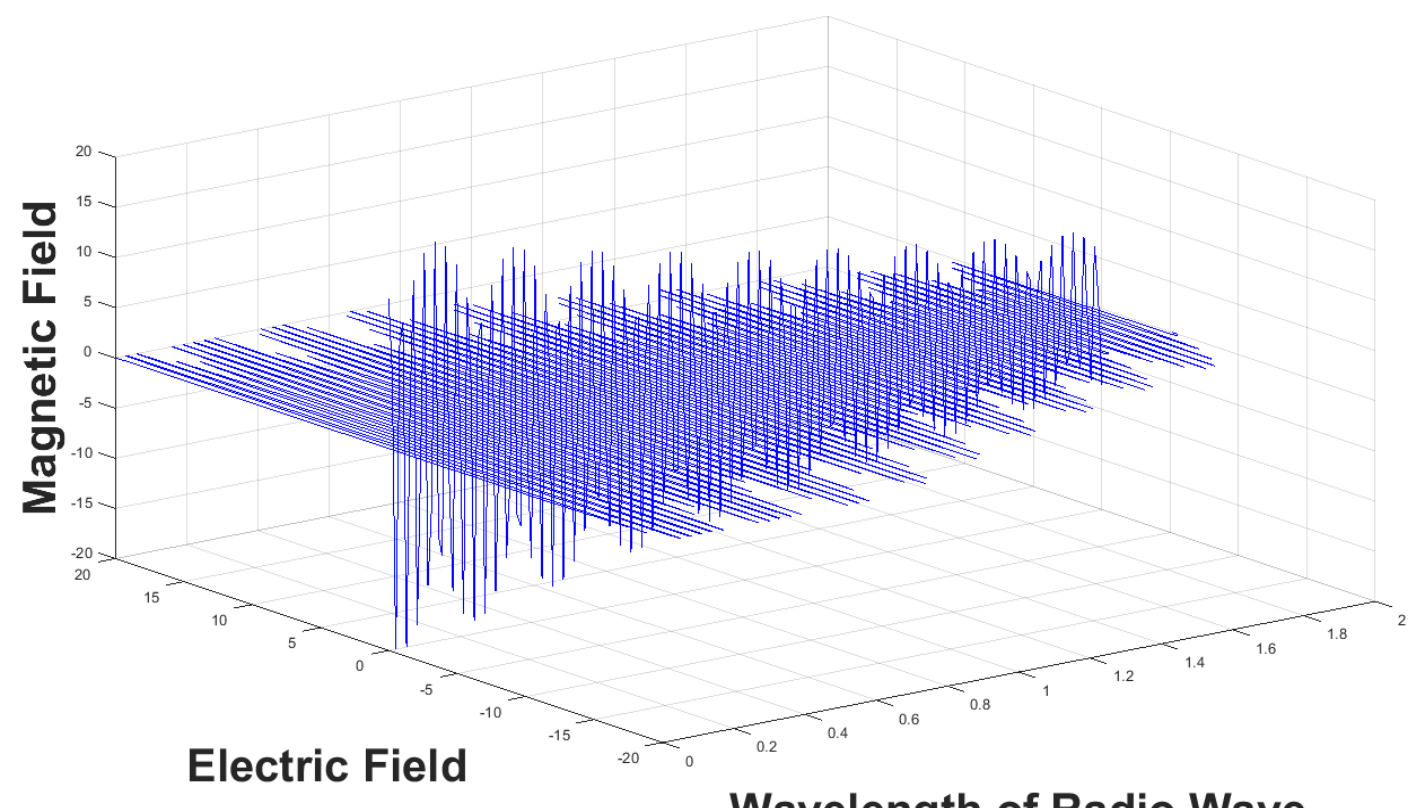

Figure 5: 5G Electromagnetic Radiation Analysis 
In Figure 5, the y axis is represented by magnetic field $\mathrm{z}$ axis is represented by electric field while the $\mathrm{x}$ axis is represented by wavelength of the radio waves. We observed that the magnetic and electric field constituents of the $5 \mathrm{G}$ radiation are transmitted at values less than zero which attenuated the electromagnetic energy that is absorbed by humans. The weak electromagnetic energy produced from emitted radiation of $5 \mathrm{G}$ wireless technology is insignificant in value to hurt human being or break apart the chemical bond of biological DNA to cause cancer diseases. However, the wavelength of the radio frequency is what gives fastest data access to users of the new technology.

In Figure 6 the biological tissue intercepted with the electromagnetic radiation of $5 \mathrm{G}$ at the value $[5,20]$ that is below the room temperature. This is also a proof that the energy generated from the $5 \mathrm{G}$ emitted waves is harmless and causes no cancer related disease because the radiation value is not enough to break the chemical bond of the human biological tissue.

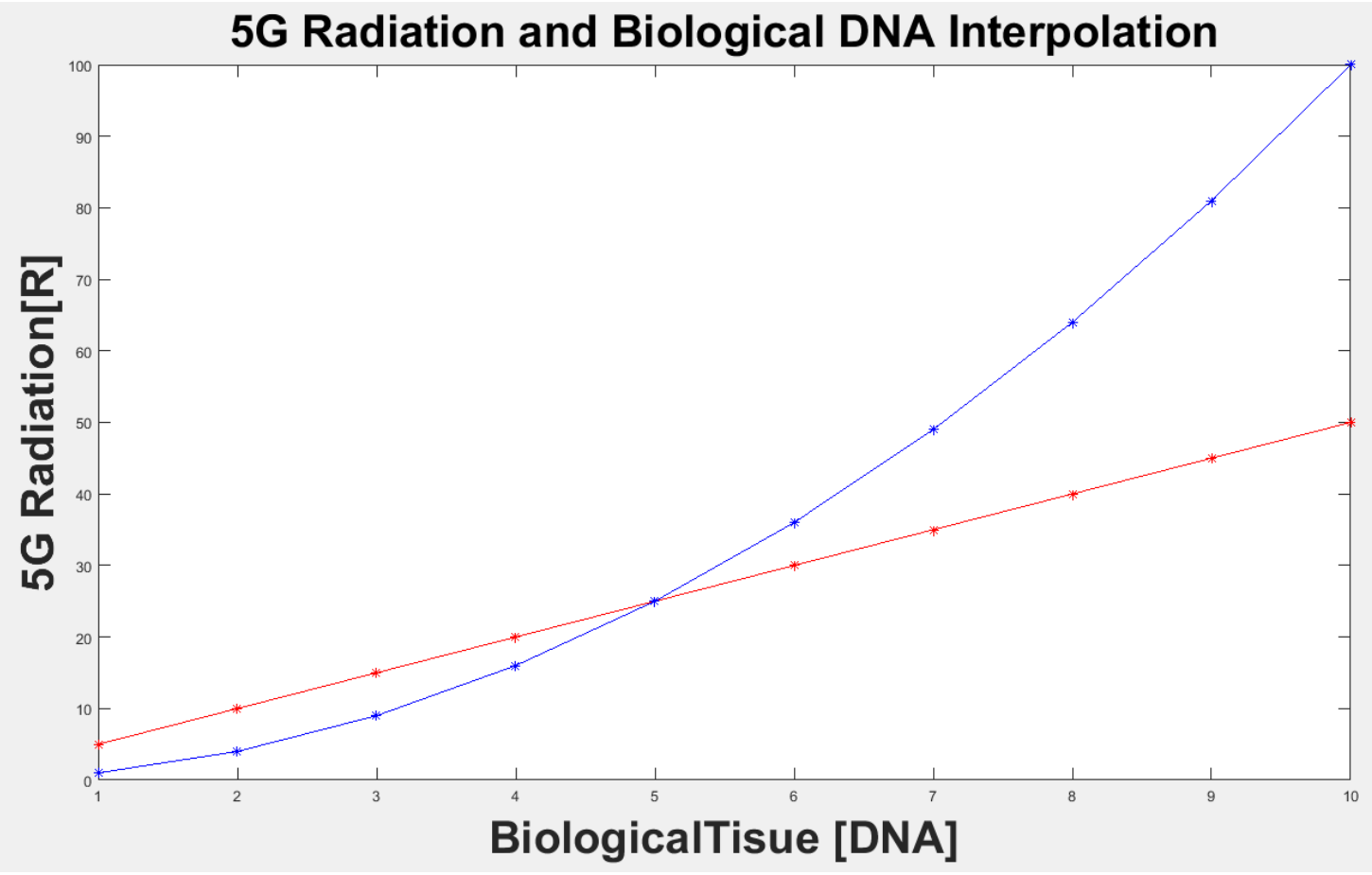

Figure 6: 5G Radiation and Biological DNA Interpolation

In this research study, with respect to result of Figure 6. The Plotted red line is designated purely for analyzing $5 \mathrm{G}$ electromagnetic radiation while the blue line is solely for the chemical bond of the DNA analysis. The interception region at $(5,20)$ is a proof that the generated temperature of $5 \mathrm{G}$ is not enough to cause any changes to cells that will lead to cancer or virus diseases.

\subsection{CONCLUSION}

In today's world, the use of internet technology is on the increase and the use of 5G is most welcoming for the fastest data access, and most reliable data transmission. With the $5 \mathrm{G}$ technology global community is miniaturized and communicates effectively without any hitch regarding the speed of data transmission and volume of devices to accommodate. Besides this positive impact, there is still fear of accepting 5G technology because of the belief that its radiation is harmful to human health. This has slowed the acceptance and spread of the new mobile wireless technology. In this research we found out that when an electromagnetic wave propagates in a conductive medium such as the human being the attenuation constant is not equal to zero, the conductivity is not zero, the propagation constant is complex, and the electromagnetic wave is attenuated during propagation. Therefore, the attenuated electromagnetic energy is incapable of breaking the chemical bonds of any biological tissue of Deoxyribonucleic Acid (DNA) or cause changes to cells that will lead to either cancer or viral-related disease. The investigated research result of this study showed that $5 \mathrm{G}$ radiation has no adverse effect on human health and we suggested that the technology should be welcomed with two hands.

\section{REFERENCES}

[1] Ruzat U., Safdar N. K.M., Arbab M.A., Salman A., Abadul H., Tariq K., and Muhammad T. "A Machine Learning Approach for 5G SINR Prediction", International Journal of Electronics, 4(2), (2020), 1-19.

[2] Magnus M. "5G positioning using Machine Learning (ML). A Thesis presented to the Department of Electrical Engineering Linkoping 
University for the award of Master of Science, (2018).

[3] Natalia Y., Yuliya G., Luis M. C., and Konstantin S. "An Analytical Model for 5G Network Resource Sharing with Flexible SLA-Oriented Slice Isolation", International Journal of Summation Mathematics, 7(4), (2020),1-19.

[4] Guto L.S., Patricia T.E., Djamel S., and Judith K. "When 5G Meets Deep Learning: A Systematic Review". International Journal of Algorithms, 6(2), (2020), 1-34.

[5] Scott F., Yuan L., Alberto P., and Stefano N. Simple Network Design and Power Allocation for 5G Device-to-Device Communication. IEEE $19^{\text {th }}$ International Workshop on Computer Aided modeling and design of Communication Links and networks (CAMAD), 8(2), (2014), 203 $-207$.

[6] Vila J, Michael C.T., Esther G.L., Jordi F., Joseph D. B., Laurel K., Lesley R., Geza B., Martine H.,
Daniel K., Dave M, Marie E.P., Siegal S., Klans S., Brigitt S.,Joachim S., Jack S., Martie, Victor T., and Elisabeth C. "Occupational exposure to high-frequency electromagnetic fields and brain tumor risk in the INTEROCC study: An individualized assessment approach", Environ Int, 8(119), (2018), 353-365.

[7] Verma S.C., Tejaswini T.M. and Devas P. "Harmful Effects of 5G Radiations: Review". Proceedings of IRAJ International Conference, $24^{\text {th }}$ March, 2019, Bengaluru, India,11(4), (2019), $71-75$.

[8] Simko M., and mattsson M. O. "5G Wireless Communication and Health Effects-A Pragmatic Review Based on available Studies regarding 6 to 100 GHZ", International Journal of Environmental Research and public Health,4(3), (2019), 1 -23. 